[21] B. Velicković, Forcing axioms and stationary sets, Advances in Math. (to appear).

[22] W. Weiss, Versions of Martin's Axiom in [12], 1984, 827-886.

\title{
Eoin Coleman
}

Department of Mathematics

College of Technology

Kevin Street

Dublin

\section{EXPLICIT RELATIONSHIPS BETWEEN ROUTH-HURWTTZ AND SCHUR-COHN TYPES OF STABILTY}

\author{
Ziad Zahreddine
}

Abstract: Given two linear systems of differential equations with real or complex coefficients, and of the same arbitrary dimension. Suppose bor in the Schur-Cohn sense. We directly express the coefficients of each in the Schur-Cohn sense. We directly express the coefficients of each plicit, make it possible to convey any stability criterion of either of the two types to the other.

\section{Introduction}

The concept of stability in differential equations has been defined in many different ways. Among these various definitions are the well-known Routh-Hurwitz and Schur-Cohn types of stability. Given a linear system of differential equations, the classical Routh-Hurwitz problem is that of obtaining necessary and sufficient conditions for all eigenvalues of the system to lie in the left half of the complex plane. The Schur-Cohn problem is that of establishing necessary and sufficient conditions for all eigenvalues to lie within the unit circle. Solutions to these problems have been the subject of intensive research over the last few years [2], $[3],[9],[12]$ and [14].

It is often noticed in the literature that some interesting results about stability, in the Hurwitz sense for example, triggers an

AMS subject classification: primary $34 \mathrm{D}$, secondary $93 \mathrm{D}$.

Key words and phrases: Routh-Hurwitz stability, Schur-Cohn stability, continuous-time systems, discrete-time systems. 
interest in the corresponding problem in the Schur sense or vice versa. See for example the introduction in [11] and example 4.2 in [8].

Recently some notable attempts have been made to give a common interpretation to the algorithms for testing the stability of continuous-time (Routh-Hurwitz) and discrete-time (SchurCohn) systems of differential equations [6], [10] and [13]. An excellent survey is given in [1] for continuous-time and in [5] for discrete-time systems.

The search for a unified approach to the study of root distribution of complex polynomials with respect to the half plane for continuous systems, and with respect to the unit disc for discrete systems, has been advocated by many eminent researchers in the field, see for example [4]. An interesting way of looking at the two problems of stability is to relate them to each other through the bilinear transformation $z=(1+w) /(1-w)$, which is equivalent to $w=(z-1) /(z+1)$. This is a one-to-one mapping between the left half of the complex $z$-plane, i.e. the region $\Re(z)<0$, and the unit disc $|w|<1$ in the complex $w$-plane. For a general discussion of bilinear transformations in this context, see [7]. Such connections prove useful in gaining new insights into the nature of the different algorithms.

This paper is a further thrust towards a firm unified approach to the relevant testing procedures for both continuous-time and discrete-time systems. In section 2 we give some notations, and the main results of the paper are given in section 3 .

\section{Notations}

If $A$ is an $n \times n$ real or complex matrix, and $X(t)$ is an $n$ dimensional column vector function of $t$, let $X^{\prime}=A X$ be system of differential equations, with eigenvalues $z_{1}, z_{2}, \ldots, z_{n}$. Then the characteristic polynomial of this system may be written in both factored and expanded forms as follows: $f(z)=$ $\prod_{j=1}^{n}\left(z-z_{j}\right)=\sum_{j=0}^{n} a_{j} z^{n-j}$ where $a_{0}=1$ by definition. Similarly if $X^{\prime}=B X$ is a system with eigenvalues $w_{1}, w_{2}, \ldots, w_{n}$ (where $w_{j}$ is related to $z_{j}$ of the previous system by $\left.w_{j}=\left(z_{j}-1\right) /\left(z_{j}+1\right)\right)$, then its characteristic polynomial is $g(w)=\prod_{j=1}^{n}\left(w-w_{j}\right)=$ $\sum_{j=0}^{n} b_{j} w^{n-j}$, with $b_{0}=1$.

The intimate relationship between Routh-Hurwitz and SchurCohn types of stability could best be expressed by the following:

Theorem 2.1. The system $X^{\prime}=A X$ is Schur-Cohn stable if and only if $X^{\prime}=B X$ is Routh-Hurwitz stable.

Proof: Suppose $z=\frac{1+w}{1-w}$, or equivalently $w=\frac{z-1}{z+1}$, where $z$ and $w$ are complex numbers. The following relationships can easily be established

$$
w+\bar{w}=\frac{2(z \bar{z}-1)}{|z+1|^{2}} \text { and } z \bar{z}-1=\frac{2(w+\bar{w})}{|1-w|^{2}},
$$

from either of which it follows that $|z|<1$ if and only if $\Re w<0$.

\section{Main Results}

If $X^{\prime}=A X$ and $X^{\prime}=B X$ are the two systems defined in section 2 with their corresponding characteristic polynomials, then

Theorem 3.1 .

$$
b_{p}=\frac{\sum_{t=0}^{n} \sum_{q=\max (t-p, 0)}^{\min (n-p, 1)}(-1)^{q}\left(\begin{array}{l}
t \\
q
\end{array}\right)\left(\begin{array}{c}
n-t \\
n-p-q
\end{array}\right) a_{t}}{\sum_{t=0}^{n}(-1)^{t} a_{t}}
$$

for all $p=1, \ldots, n$.

Proof: Consider $f(z)=\sum_{t=0}^{n} a_{t} z^{n-t}$ with zeros $z_{1}, \ldots, z_{n}$ and $z_{j}=\left(1+w_{j}\right) /\left(1-w_{j}\right)$ for $j=1, \ldots, n$. Hence $w_{1}, \ldots, w_{n}$ are the zeros of

$$
\begin{aligned}
f\left(\frac{1+w}{1-w}\right) & =\sum_{t=0}^{n} a_{t}\left(\frac{1+w}{1-w}\right)^{n-t} \\
& =\frac{1}{(1-w)^{n}} \sum_{t=0}^{n} a_{t}(1-w)^{t}(1+w)^{n-t}
\end{aligned}
$$


Therefore $w_{1}, \ldots, w_{n}$ are the zeros of the polynomial

$$
\begin{aligned}
\dot{h}(w) & =\sum_{t=0}^{n} a_{t}(1-w)^{t}(1+w)^{n-t} \\
& =\sum_{t=0}^{n} a_{t} \sum_{r=0}^{t}(-1)^{r}\left(\begin{array}{c}
t \\
r
\end{array}\right) w^{r} \sum_{s=0}^{n-t}\left(\begin{array}{c}
n-t \\
s
\end{array}\right) w^{s} \\
& =\sum_{t=0}^{n} \sum_{r=0}^{t} \sum_{s=0}^{n-t}(-1)^{r}\left(\begin{array}{c}
t \\
r
\end{array}\right)\left(\begin{array}{c}
n-t \\
s
\end{array}\right) a_{t} w^{r+s} .
\end{aligned}
$$

We make the following transformation from the $(r, s)$ plane to the $(p, q)$ plane:

$$
p=n-r-s, q=r .
$$

Then the rectangle in the $(r, s)$ plane with sides $r=0, r=t, s=0$, $s=n-t$ is transformed into the parallelogram in the $(p, q)$ plane with sides $q=0, q=t, q=n-p$, $q=t-p$. Hence

$$
h(w)=\sum_{t=0}^{n} \sum_{p=0}^{n} \sum_{q=\max (t-p, 0)}^{\min (n-p, t)}(-1)^{q}\left(\begin{array}{l}
t \\
q
\end{array}\right)\left(\begin{array}{c}
n-t \\
n-p-q
\end{array}\right) a_{i} w^{n-p}
$$

Wrice $h(i w)=\sum_{p=0}^{n} N_{p} w^{n-p}$, where

$$
N_{p}=\sum_{t=0}^{n} \sum_{q=\max (t-p, 0)}^{\min (n-p, t)}(-1)^{q}\left(\begin{array}{l}
t \\
q
\end{array}\right)\left(\begin{array}{c}
n-t \\
n-p-q
\end{array}\right) a_{t}
$$

In the polynomial $h(w)$, the leading coefficient is

$$
N_{0}=\sum_{t=0}^{n}(-1)^{t}\left(\begin{array}{l}
t \\
t
\end{array}\right)\left(\begin{array}{l}
n-t \\
n-t
\end{array}\right) a_{t}=\sum_{t=0}^{n}(-1)^{t} a_{t}
$$

Now the two polynomials

$$
\frac{1}{N_{0}} h(w)=\sum_{p=0}^{n} \frac{N_{p}}{N_{0}} w^{n-p} \quad \text { and } g(w)=\sum_{p=0}^{n} b_{p} w^{n-p}
$$

being both monic and having the same set of zeros are identical, leading automatically to the desired conclusion.

The converse of Theorem 3.1 states the following

Theorem 3.2.

$$
a_{p}=\frac{\sum_{t=0}^{n} \sum_{q=\max (t-p, 0)}^{\min (n-p, t)}(-1)^{p+q-1}\left(\begin{array}{l}
t \\
q
\end{array}\right)\left(\begin{array}{c}
n-t \\
n-p-q
\end{array}\right) b_{t}}{\sum_{t=0}^{n} b_{t}}
$$

for all $p=1, \ldots, n$.

The proof of this theorem is omitted as it is similar to that of Theorem 3.1.

The author wishes to thank the referee for many valuable suggestions which have led to significant improvements of the paper.

\section{References}

[1] B. R. Barmish and C. L. DeMarco, Criteria for robust stability with structured uncertainty: a perspective, Proc. Amer. Cont. Conf., Minneapolis, MN (1987).

[2] M. Benidir and B. Picinbono, Wide sense stability of discrete- and continuous-time linear systems in the complex case, IEEE Trans. Acoust. Speech Signal Process. 37(No 11) (1989), 1768-1771.

[3] Y. Bistritz, Stability criterion for continuous-time system polynomials with uncertain complex coefficients, IEEE Trans. Circuits and Systems 35(No 4) (1988), 442-450. 
[4] N. K. Bose and Y. Q. Shi, Network realizability theory approach to stability of complex polynomials, IEEE Trans. Circuits and Systems 34(No 2) (1987), 216-218.

[5] N. K. Bose and E. Zeheb, Kharitonov's theorem and stabillity test of multidimensional digital filters, Proc. IEE-G 133 (1986), 187-190.

[6] P. Delsarte, Y. Genin and Y. Kamp, Pseudo-lossless functions with application to the problem of locating the zeros of a polynomial, IEEE Trans. Circuits and Systems 32 (1985), 373-381.

[7] C. V. Hollot and A. C. Bartlet, Some discrete-time counterparts to Kharitonov's stability criterion for uncertain systems, IEEE Trans. Automat. Control 31 (No 4) (1986), 355-356.

[8] E. I. Jury and A. Katbab, A note on Kharitonov-type results in the space of Markov parameters, IEEE Trans. Automat. Control 37(No 1) (1992), 155-158.

[9] A. Katbab, F. Kraus and E. I. Jury, Some Schur-stability criteria for uncertain systems with complex coefficients, IEEE Trans. Circuits and Systems 37 (No 9) (1990), 1171-1176.

[10] W. Krajewski, A. Lepschy, G. A. Mian and U. Viaro, A unifying frame for stability-test algorithms for continuous-time systems, IEEE Trans. Circuits and Systems 37(No 2) (1990), 290-296.

[11] F. Kraus, M. Mansour and E. I. Jury, Robust Schur stability of interval polynomials, IEEE Trans. Automat. Control 37(No 1) (1992), 141143.

[12] I. R. Petersen, A class of stability regions for which a Kharitonovlike theorem holds, IEEE Trans. Automat. Control 34(No 10) (1989), $1111-1115$.

[13] P. P. Vaidyanathan and S. K. Mitra, A unified structural interpretation for some well-known stability-test procedures for linear systems, Proc. IEEE 75 (1987), 478-497.

[14] Z. Zahreddine, An extension of the Routh array for the asymptotic stability of a system of differential equations with complex coefficients, Appl. Anal. (to appear)

Department of Mathematics

Faculty of Sciences

U. A. E. University

Al-Ain

P. O. Box 17551

United Arab Emirates

\section{UNDERGRADUATE PROJECTS}

S. K. Houston and N. H. Smith

Abstract: The rationale for, operation of and assessment of undergraduate projects at the University of Ulster are discussed. Specimen project titles are provided.

\section{Introduction}

A debate on undergraduate mathematics teaching in Ireland has recently been started through this Bulletin [1], [2]. It has been continued at a conference organized by the Sub-Commission for Mathematical Instruction of the Royal Irish Academy and held in Dublin in September 1991 (RIA-91), [3].

O'Reilly [1] questioned how we teach mathematics at tertiary level, leaving readers with many "focusing questions" and "questions for exploration". Dickenson et al. [2] described innovative methods of teaching, learning and assessment used at the University of Ulster, and went some way to answering O'Reilly's questions. Ted Hurley (UCG) continued the debate in his plenary lecture "Mathematics at Third Level" at RIA-91. In his lecture he pointed out that

(i) the number of honours graduates in mathematics from Irish Universities per capita is 3.5 times smaller than the number per capita from British Universities;

(ii) $45 \%$ of these Irish graduates entered further study compared to $14.5 \%$ of British graduates.

He concluded that this was an unsatisfactory state of affairs and made some suggestions for remedying the situation such as putting greater emphasis on the links between mathematics and computing. 